Pacific Journal of Mathematics

SOME REMARKS ON VARIETIES IN POLYDISCS AND
BOUNDED HOLOMORPHIC FUNCTIONS 


\title{
SOME REMARKS ON VARIETIES IN POLYDISCS AND BOUNDED HOLOMORPHIC FUNCTIONS
}

\author{
E. L. STOUT
}

This note deals with certain questions which arise in connection with the extension problem for bounded holomorphic functions of several complex variables.

An analytic variety $V$ in the unit polydisc

$$
U^{N}=\left\{\left(z_{1}, \cdots, z_{N}\right) \in \mathbf{C}^{N}:\left|z_{1}\right|, \cdots,\left|z_{N}\right|<1\right\}
$$

is said to have the $H^{\infty}$-extension property if for every $f \in H^{\infty}(V)$, the space of bounded holomorphic functions on $V$, there is $F \in H^{\infty}\left(U^{N}\right)$ such that $F \mid V=f$. A related property which $V$ may possess is that of being defined as a set by bounded functions in the sense that

$$
V=\left\{z \in U^{N}: f(z)=0 \text { for all } f \in H^{\infty}\left(U^{N}\right) \text { which vanish on } V\right\} \text {. }
$$

We will begin with an example of a remarkably well behaved variety $V \subset U^{N}$ which fails to have the $H^{\infty}$-extension property, and after this we give an example of a one dimensional disc $D$ embedded as a submanifold of $U^{N}$ which not only fails to be defined as a set by bounded functions but is, in fact, a determining set for $H^{\infty}\left(U^{N}\right)$ in that if $f \in H^{\infty}\left(U^{N}\right)$ vanishes on $D$, then $f$ is the zero function. Positive results obtained include a geometric condition for a $U^{k}$ embedded in a $U^{N}$ to be defined as a set by bounded functions and a result to the effect that if a variety $V$ has the $H^{\infty}$-extension property and if it satisfies another, possibly redundant, condition, then $V$ is defined as a set by bounded functions.

The general problem of determining which subvarieties of $U^{N}$ possess the $H^{\infty}$-extension property seems to be difficult, but some results in this direction are contained in the papers [1] and [9].

We begin with an example of a disc contained in $U^{2}$ which does not have the $H^{\infty}$-extension property.

Example 1. Denote by $\mathbf{C}^{*}$ the Riemann sphere and in $\mathbf{C}^{*}$ let

$$
\Delta=\left\{\zeta:\left|1-\zeta^{2}\right|^{-1}<1\right\} \text {. }
$$

The set $\Delta$ is conformally equivalent to $U$. If we define $\Phi: \mathbf{C}^{*} \backslash\{1,-1\} \rightarrow \mathbf{C}^{2}$ by $\Phi(\zeta)=\left(\left(1-\zeta^{2}\right)^{-1}, \varepsilon(1-\zeta)^{-1}\right)$, then for all choices of $\varepsilon>0$, the mapping $\Phi$ carries $C^{*} \backslash\{1,-1\}$ biholomorphically onto a closed, algebraic submanifold $M$ of $C^{2}$, and if $\varepsilon$ is small enough, then $M \cap U^{2}=\Phi(\Delta)$ so that $\Phi(\Delta)$ is a disc embedded in $U^{2}$. Assume that $\Phi(\Delta)$ has the 
$H^{\infty}$-extension property so that if $f \in H^{\infty}(\Delta)$, there is $F \in H^{2}\left(U^{2}\right)$ such that $f=F \circ \Phi$. Let $h: \Delta \rightarrow U$ be a conformal homeomorphism chosen so that $\lim _{y \rightarrow 0^{+}} h(i y)=1$ and $\lim _{y \rightarrow 0^{-}} h(i y)=-1$. Our assumption on $\Phi(\Delta)$ implies that if $\delta>0$ is small enough, then there exists $F \in H^{\infty}\left(U^{2}\right)$ such that $\|F\|_{U^{2}} \leqq 1$ and $F(\Phi(\zeta))=\delta h(\zeta)$ for all $\zeta \in \Delta$. In particular

$$
\lim _{y \rightarrow 0^{+}} F(\Phi( \pm i y))= \pm \delta \text {. }
$$

If $z$ and $w$ are points of $U$, set

$$
[z, w]=\left|\frac{z-w}{1-\bar{w}_{z}}\right| \text {. }
$$

By the invariant form of Schwarz's lemma, we know that if $g$ lies in the unit ball of $H^{\infty}(U)$, then

$$
[g(z), g(w)] \leqq[z, w] .
$$

If we apply (2) to the function $h_{\eta}$ given for fixed $\eta \in U$ by $h_{\eta}(\zeta)=$ $F(\eta, \zeta)$, then for $\eta=\left(1-(i y)^{2}\right)^{-1}, z=\varepsilon /(1-i y), w=\varepsilon /(1+i y)$, we are led to

$$
\lim _{y \rightarrow i^{+}}[F(\Phi(i y)), F(\Phi(-i y))] \leqq \lim _{y \rightarrow 0^{+}}\left[\frac{\varepsilon}{1-i y}, \frac{\varepsilon}{1+i y}\right]=0 .
$$

However, (1) implies that

$$
\lim _{y \rightarrow 0^{+}}[F(\Phi(i y)), F(\Phi(-i y))]=\frac{2 \delta}{1+\delta^{2}} .
$$

This contradiction shows that the disc $\Phi(\Delta)$ does not have the $H^{\infty}$ extension property.

The present example is not the first known instance of this phenomenon; another, more involved example was given in [9, Example II. 7]. It can be show, at least for certain choices of the Blaschke product involved, that example has the additional property of being a determining set for $H^{\infty}\left(U^{2}\right)$. The variety $\Phi(\Delta)$ of the present example is not nearly so pathological, for it is the intersection of $U^{2}$ with the zero set of a certain polynomial in two variables. Alexander [1] has also given an example of a variety in $U^{2}$ which lacks the $H^{\infty}$-extension property. His example is the intersection of $U^{2}$ with a certain algebraic curve, but it is not irreducible. In [8] Rudin has also given an example.

In connection with Example 1, it is interesting to consider the composition $\Phi \circ \psi$ where $\psi$ is a conformal homeomorphism from the unit disc to $\Delta$ such that $\psi(0)=\infty$. If we let $\varphi_{1}(\zeta)=\left(1-\zeta^{2}\right)^{-1}$, then $\varphi_{1} \circ \psi$ is a two-to-one map from the disc onto itself, and it can be 
verified without difficulty that $\varphi_{1} \circ \psi(z)=\alpha z^{2}$ for some $\alpha$ of modulus one. Also, if $\varphi_{2}(\zeta)=\varepsilon(1-\zeta)^{-1}$, then $\varphi_{2} \circ \psi$ is one-to-one from the disc into itself, and it is not hard to see that if $z_{0}$ and $z_{1}$ are the two points in the unit circle carried onto 0 by $\psi$, then $\varphi_{2} \circ \psi$ continues across the two arcs of the unit circle determined by $z_{0}$ and $z_{1}$. These two points are certain algebraic singularities of the function $\varphi_{2} \circ \psi$. These remarks should be compared with the extension theorems for bounded holomorphic functions proved in [9] and [10]; they show that those extension theorems are essentially the best of their kind.

EXAMPLE 2. In this example we will construct in $U^{N}, N \geqq 2$, a disc which is a determining set for $H^{\infty}\left(U^{N}\right)$.

Let $\Omega=U \backslash[0,1)$, and let $h: U \rightarrow \Omega$ be a conformal homeomorphism which takes 1 to $1, i$ to 0 and which has the property that $\operatorname{Im} h(\zeta) \downarrow 0$ as $\zeta \rightarrow e^{i \theta}$ if $\theta \in(0, \pi / 2)$. The function $h$ admits a unique extension to a continuous function from $\bar{U}$ to $\bar{U}$.

Let $r_{k}=1-k^{-1}$, and let $s_{1}>0$ be very small so that $\left\{r_{k}+i s_{k}\right\}$ does not satisfy the Blaschke condition, i.e., this sequence is not the zero set of a function bounded and holomorphic in $U$. If $\left\{s_{k}\right\}$ is chosen properly and if $\alpha_{k}=h^{-1}\left(r_{k}+i s_{k}\right)$, the sequence $\left\{\alpha_{k}\right\}$ will satisfy the Blaschke condition. Let $B$ be the Blaschke product with $\left\{\alpha_{k}\right\}$ as its zero set, and define $\Phi$ by $\Phi(\zeta)=(h(\zeta), B(\zeta))$. The sequence $\left\{\alpha_{k}\right\}$ converges to the point 1 , so it follows that at every point of $\partial U$, either $|B|$ or $|h|$ assumes continuously the value 1 . Since $h^{\prime}$ is zero-free and $h$ is one-to-one, it follows that $\Delta=\Phi(U)$ is an analytic submanifold of $U^{2}$.

We will prove that if $F \in H^{\infty}\left(U^{2}\right)$ and $F \circ \Phi=0$, then $F$ is the zero function, i.e., that $\Delta$ is a determining set for $H^{\infty}\left(U^{2}\right)$. If $F \in H^{\infty}\left(U^{2}\right)$ vanishes on $\Phi(U)$, then $F\left(r_{k}+i s_{k}, 0\right)=0$ for all $k$, so since $\left\{r_{k}+i s_{k}\right\}$ does not satisfy the Blaschke condition, $F$ must vanish identically on the disc $D=\{(z, 0):|z|<1\}$. If $F$ does not vanish identically, there is a factorization $F(z, w)=w^{p} G(z, w)$ where $p$ is a positive integer and $G$ a bounded holomorphic function which does not vanish identically on $D$. As $F$ vanishes on $\Phi(U), G$ must also. This implies, as we have just seen, that $G\left(r_{k}+i s_{k}, 0\right)=0$ whence $G$ vanishes on the disc $D$, contrary to hypothesis.

Thus we have a disc in $U^{2}$ which is a determining set for $H^{\infty}\left(U^{2}\right)$. It is quite simple, using the existence of this disc, to find a disc in $U^{N}, N \geqq 2$, which is a determining set for $H^{\infty}\left(U^{N}\right)$. We proceed inductively. Suppose that $\Delta \subset U^{k}$ is a disc which is a determining set for $H^{\infty}\left(U^{k}\right)$. Then $U^{k+1}=U^{k} \times U \supset \Delta \times U$. The set $\Delta \times U$ is biholomorphically equivalent to $U^{2}$ so there is a one dimensional disc $\Delta^{\prime}$ which is a determining set for $\Delta \times U$. Suppose that $F \in H^{\infty}\left(U^{k+1}\right)$ vanishes on $\Delta^{\prime}$. If we take on $U^{k+1}$ the coordinates $(z, \zeta), z \in U^{k}, \zeta \in U$, then since $\Delta^{\prime}$ is a determining set for $\Delta \times U$, it follows that for each 
$\zeta \in U, F(\cdot, \zeta)$ vanishes identically. As this holds for every $\zeta \in U, F$ must be the zero function.

Our next example is a direct consequence of the construction given in Example 2.

EXAMPLE 3. In $U^{N}, N \geqq 3$, there exist irreducible varieties $V$ which are at positive distance, in the sense of the usual metric on $C^{N}$, from the distinguished boundary, $T^{N}$, of $U^{N}$ and yet which are not defined, as sets, by bounded functions. To optain such an example, let $\Delta$ be an irreducible variety, e.g., a disc, which is a determing set for $H^{\infty}\left(U^{2}\right)$. The set $\Delta \times\{0\} \subset U^{2} \times U^{N-2}=U^{N}$ is an example of a variety of the desired kind.

This example is of interest because it contracts markedly with a theorem of Rudin [7] according to which if $V \subset U^{N}$ is a variety of codimension 1 which is at positive distance from $T^{N}$, then not only is $V$ defined as a set by a single bounded function, but, in addition, there is an $F \in H^{\infty}\left(U^{N}\right)$ with the property that every function holomorphic in $U^{N}$ and vanishing on $V$ admits a factorization $G=F H, H$ holomorphic in $U^{N}{ }^{1}$

Our next result gives a sufficient condition for a disc or polydisc contained in $U^{N}$ to be defined, as a set, by bounded holomorphic functions.

THEOREM 4. Let $\Phi: U^{k} \rightarrow U^{N}$ be a proper, holomorphic map, $k \leqq N$, say $\Phi(\xi)=\left(\varphi_{1}(\xi), \cdots, \varphi_{N}(z)\right)$. If there is a $\delta>0$ with the property that for each $z \in U^{k}$ at least $N-k$ of $\left|\varphi_{1}(z)\right|, \cdots,\left|\varphi_{N}(z)\right|$ are no more than $1-\delta$, then the variety $\Phi\left(U^{k}\right)$ is defined as a set by bounded holomorphic functions.

Let us remark that since $\Phi$ is proper, $\Phi\left(U^{k}\right)$ is a variety by [4, Th. V.C. 5].

Proof. Consider first the case that $k=1$. Let

$$
K=\left\{\left(z_{1}, \cdots, z_{N}\right) \in U^{N}:\left|z_{1}\right|, \cdots,\left|z_{N}\right| \leqq 1-\delta\right\} .
$$

The set $\Phi^{-1}(K)$ is compact, and by the maximum modulus theorem no component of the set $\Sigma=U \backslash \Phi^{-1}(K)$ can be bounded away from $\partial U$, so $\Sigma$ is connected. If $\zeta \in \Sigma$, then for some $j,\left|\varphi_{j}(\zeta)\right|>1-\delta$. Let

$$
\Sigma_{j}=\left\{\zeta \in \Sigma:\left|\varphi_{j}(\zeta)\right|>1-\delta\right\} \text {. }
$$

${ }^{1}$ Added in proof. Y.-T. Siu in his paper Sheaf cohomology with bounds and bounded holomorphic functions, Proc. Amer. Math. Soc. 21 (1969), 226-229, has given a cohomological proof of this and a related result. 
The sets $\Sigma_{j}$ are all open, they are pairwise disjoint, and their union is the connected set $\Sigma$. Thus one of them, say $\Sigma_{1}$, is the whole of $\Sigma$ and all the other $\Sigma_{j}$ are empty. The map $\Phi$ is proper so it follows that $\left|\varphi_{1}(\zeta)\right| \rightarrow 1$ as $|\zeta| \rightarrow 1$. (Although we do not need this fact, it follows that $\varphi_{1}$ is a finite Blaschke product.)

Now consider the case of general $k$. By [8, Th. II. 3], we may reindex the functions $\varphi_{1}, \cdots, \varphi_{N}$, so that if $z=\left(z_{1}, \cdots, z_{N}\right)$, then for $1 \leqq j \leqq k, \varphi_{j}(z)$ depends only on $z_{j}$ and so that if

$$
\varphi_{j}^{*}\left(e^{i \theta}\right)=\lim _{r \rightarrow 1^{-}} \varphi_{j}\left(r e^{i \theta}\right),
$$

then $\left|\varphi_{j}^{*}\left(e^{i \theta}\right)\right|=1$ on a set of $\theta$ 's of positive measure. It follows that we can choose $z_{2}^{0}, \cdots, z_{k}^{0}$, of modulus less than one so that for some $\eta, 1>\eta>\left|\varphi_{j}\left(z_{j}^{0}\right)\right|>1-\delta$. Define $\psi: U \rightarrow U^{k}$ by

$$
\psi(\zeta)=\left(\zeta, z_{2}^{0}, \cdots, z_{k}^{0}\right)
$$

The map $\psi$ is proper, so $\Phi \circ \psi$ is a proper map from $U$ into $U^{N}$. We have that of the $N$ coordinates of $\Phi(\psi(\zeta)), k-1$, viz., $\varphi_{2}\left(z_{2}^{0}\right), \cdots, \varphi_{k}\left(z_{k}^{0}\right)$, exceed $1-\delta$, so by our hypothesis on $\Phi$, at least $N-k$ of $\left|\varphi_{1}(\zeta)\right|$, $\left|\varphi_{k+1}(\psi(\zeta))\right|, \cdots,\left|\varphi_{N}(\psi(\zeta))\right|$ are less than $1-\delta$. Thus the map $\Phi \circ \psi$ has the property that if $\Phi \circ \psi(\zeta)=\left(w_{1}, \cdots, w_{N}\right)$, then at least $N-1$ of $\left|w_{1}\right|, \cdots,\left|w_{N}\right|$ are less than $\eta$. By our consideration of the case $k=1$, it follows that one of $\left|\varphi_{1}\right|,\left|\varphi_{k+1} \circ \psi\right|, \cdots,\left|\varphi_{N} \circ \psi\right|$, tends to one at the boundary of the unit disc while the others remain bounded away from one. As $\left|\varphi_{1}^{*}\right|=1$ on a set of positive measure, we may conclude that $\left|\varphi_{1}\left(z_{1}\right)\right| \rightarrow 1$ as $\left|z_{1}\right| \rightarrow 1$. In the same way we can show that $\left|\varphi_{j}\left(z_{j}\right)\right| \rightarrow 1$ as $\left|z_{j}\right| \rightarrow 1,2 \leqq j \leqq k$.

Let $\pi: U^{N} \rightarrow U^{k}$ be the natural projection onto the first $k$ coordinates, and set $V=\Phi\left(U^{k}\right)$. We know that $V$ is a variety, and what we have done implies that $\pi$ carries $V$ properly onto $U^{k}$. Thus the triple $\left(V, \pi \mid V, U^{k}\right)$ is an analytic cover so our result is a consequence of the following general fact.

Lemma 5. Let $\Omega \subset \mathrm{C}^{m}$ and $\Omega^{\prime} \subset \mathrm{C}^{n}$ be bounded domains, let $V \subset$ $\Omega \times \Omega^{\prime}$ be a purely $m$ dimensional variety, and let $\pi: \Omega \times \Omega^{\prime} \rightarrow \Omega$ be the natural projection. If $\left(V, \pi \mid V, \Omega^{\prime}\right)$ is an analytic cover, then $V$ is defined as a set by bounded holomorphic functions on $\Omega \times \Omega^{\prime}$.

This lemma is contained in the proof of [4, III. B. 19].

We finish with a result which partially-only partially-answers an obvious question: If the variety $V \subset U^{N}$ has the $H^{\infty}$-extension property, does it necessarily follow that $V$ is defined as a set by bounded holomorphic functions? It seems probable that this question has an 
affirmative answer without qualification on the variety $V$, but we are able to prove a result in this direction only by making an additional assumption.

THEOREM 6. If $V \subset U^{N}$ is a variety with the $H^{\infty}$-extension property and if $V$ is open in the spectrum of $H^{\infty}(V)$, then $V$ is defined as a set by bounded holomorphic functions.

We understand by the spectrum of a commutative Banach algebra $A$ the space consisting of the nonzero complex homomorphisms of $A$ taken with the weak* topology. We denote the spectrum of $A$ by $\Sigma(A)$.

Proof. We define an ideal $I^{\infty}(V)$ and a variety $\widetilde{V}$ by

$$
I^{\infty}(V)=\left\{f \in H^{\infty}\left(U^{N}\right): f \text { vanishes on } V\right\}
$$

and

$$
\widetilde{V}=\left\{z \in U^{N}: f(z)=0 \text { for all } f \in I^{\infty}(V)\right\} .
$$

The variety $\widetilde{V}$ evidently contains $V$ and we will prove, under the hypotheses of the theorem, that $\widetilde{V}=V$. The restriction map $\rho$ from $H^{\infty}\left(U^{N}\right)$ to $H^{\infty}(V)$ is onto and consequently $\Sigma\left(H^{\infty}(V)\right)$ can be identified with the set

$$
\left\{\phi \in \Sigma\left(H^{\infty}\left(U^{N}\right)\right): \varphi f=0 \text { if } f \in I^{\infty}(V)\right\} .
$$

This set contains $\widetilde{V}$ in a natural way and as $V$ is assumed to be open in $\Sigma\left(H^{\infty}(V)\right)$, it follows that $V$ is an open subset of $\tilde{V}$. Plainly, $V$ is closed in $\tilde{V}$.

As $\widetilde{V} \supset V$, the hypotheses of the theorem imply that the restriction map $\rho^{\prime}$ from $H^{\infty}(\widetilde{V})$ to $H^{\infty}(V)$ is onto so we can identify $\Sigma\left(H^{\infty}(V)\right)$ with

$$
\left\{\varnothing \in \Sigma\left(H^{\infty}(\tilde{V})\right) ; \phi f=0 \quad \text { if } f \in \operatorname{ker} \rho^{\prime}\right\} .
$$

The characteristic function $\chi$ of $\tilde{V} \backslash V$ lies in $H^{\circ}(\tilde{V})$ since $V$ is open and closed in $\tilde{V}$. Since $\chi \in \operatorname{ker} \rho^{\prime}$, it follows that $\widetilde{V} \backslash V$ cannot meet $\Sigma\left(H^{\infty}(V)\right)$. We know that $\widetilde{V} \subset \Sigma\left(H^{\infty}(V)\right)$ so we conclude that $\widetilde{V}=V$ as was to be proved.

Our formulation of Theorem 6 suggests another question: If $V$ is an analytic variety, is it open in $\Sigma\left(H^{\infty}(V)\right)$ ? This question does not seem to have an obvious answer even for subvarieties of a polydisc though it does seem likely that generally $V$ is open in $\Sigma\left(H^{\infty}(V)\right)$. The following remarks are relevant.

REMARKs 7. (a) It is well known that the unit disc is open in $\Sigma\left(H^{\infty}(U)\right)$. (See [5].) Similarly, $U^{N}$ is open in $\Sigma\left(H^{\infty}\left(U^{N}\right)\right)$. 
(b) It is not hard to see that for many familiar open sets $S$ in $C^{N}$, e.g., balls, special analytic polyhedra [4], $S$ is open in $\Sigma\left(H^{\infty}(S)\right)$.

(c) For general one dimensional varieties $V$, we do not know that $V$ is open in $\Sigma\left(H^{\infty}(V)\right)$, but the following rather ad hoc argument settles the question for certain Riemann surfaces. Let $R$ be an open connected Riemann surface of finite genus so that $R=R_{1} \backslash E, R_{1}$ a compact Riemann surface and $E$ a closed subset thereof. If $H^{\infty}(R)$ contains a nonconstant function, then $R$ is open in $\Sigma\left(H^{\infty}(R)\right)$. Since $R$ is contained in a compact surface and $H^{\infty}(R)$ contains a nonconstant function, it follows easily from the Riemann-Roch theorem that $H^{\infty}(R)$ separates points on $R$. (In the case that $R_{1}$ is of genus zero, this sort of result is in papers of Rudin [6] and Wermer [11]; the case of general, finite, genus follows in an analogous way.)

Let $\zeta_{0} \in R . \quad B y$ the Riemann-Roch theorem there exists a function $h$ meromorphic on the ambient surface $R_{1}$ which has only one pole, that at $\zeta_{0}$ and of assigned order $p$ if $p$ is large enough. Thus, for a suitable function $h_{1} \in H^{\infty}(R)$, the function $H=h h_{1}$ will have at $\zeta_{0}$ a simple pole, it will be holomorphic on $R \backslash\left\{\zeta_{0}\right\}$, and it will be bounded off a neighborhood of $\zeta_{0}$. Define an operator $T: H^{\infty}(R) \rightarrow H^{\infty}(R)$ by

$$
T(f)=\left(f-f\left(\zeta_{0}\right)\right) H .
$$

The properties of the function $H$ show that $T$ is a bounded linear operator on $H^{\infty}(R)$ and that

$$
T(f g)=g T(f)+f\left(\zeta_{0}\right) T(g) .
$$

Thus in the terminology of Banaschewki [3], $T$ is a bounded derivation of type $\left(I, \zeta_{0}\right)$. By Proposition 1 of [3], a result previously obtained by Bishop [2], there is a homeomorphism $\Phi$ from the open unit disc $U$ onto an open set in $\Sigma\left(H^{\infty}(R)\right)$ such that $\Phi(0)=\zeta_{0}$ and such that if $f \in H^{\infty}(R)$, then $\hat{f} \circ \Phi$ is holomorphic on $U$. Since there is a disc in $R$ through $\zeta_{0}$, it follows from the openness of $\Phi(U)$ in $\Sigma\left(H^{\infty}(R)\right)$ that some neighborhood of $\zeta_{0}$ in $R$ is at the same time a neighborhood of $\zeta_{0}$ in $\Sigma\left(H^{\infty}(R)\right)$. It follows that $R$ is open in $\Sigma\left(H^{\infty}(R)\right)$ as was to be proved.

We are indebted to the referee whose suggestions have led to material simplifications of several points in the paper.

\section{REFERENCES}

1. H. Alexander, Extending bounded holomorphic functions from certain subvarieties of a polydisc, Pacific J. Math. 29 (1969), 485-490.

2. E. Bishop, Analyticity in certain Banach algebras, Trans. Amer. Math. Soc. 102 (1962), 507-544. 
3. B. Banaschewski, Analytic discs in the maximal ideal space of a Banach algebra, Bull. Acad. Polonaise des Sciences, Ser. Math. Astro., Phy. 14 (1966), 137-144.

4. R. C. Gunning and H. Rossi, Analytic functions of several complex variables, Prentice-Hall, Englewood Cliffs, 1965.

5. K. Hoffman, Banach spaces of analytic functions, Prentice-Hall, Englewood Cliffs, 1962.

6. W. Rudin, Subalgebras of spaces of continuous functions, Proc. Amer. Math. Soc. 7 (1956), 825-830.

7. _ Zero-sets in polydiscs, Bull. Amer. Math. Soc. 73 (1967), 580-83.

8. _ Function theory in polydiscs, W. A. Benjamin, Inc., New York, 1969.

9. W. Rudin and E. L. Stout, Modules over polydisc algebras, Trans. Amer. Math. Soc. 138 (1969), 327-342.

10. E. L. Stout, On some algebras of analytic functions on finite open Riemann surfaces, Math. Zeit. 92 (1966), 366-379.

11. J. Wermer, Polynomial approximation on an arc in $C^{3}$, Ann. of Math. (2) 62 (1955), 269-270.

Received January 6, 1969. The research for this paper was started at the University of Washington under the auspices of an Office of Naval Research Associateship, and it was concluded at Yale with support from N.S.F. Grant GP-9049.

YALE UNIVERSITY

UNIVERSITY OF WASHINGTON 


\title{
PACIFIC JOURNAL OF MATHEMATICS
}

\author{
EDITORS
}

H. SAMELSON

Stanford University

Stanford, California 94305

J. DugunduI

Department of Mathematics

University of Southern California

Los Angeles, California 90007

RICHARD PIERCE

University of Washington

BASIL GORDON*

University of California

Seattle, Washington 98105

Los Angeles, California 90024

\section{ASSOCIATE EDITORS}

E. F. BeCKenbaCh

B. H. NeumanN

F. WOLF

K. YosHIDA

\section{SUPPORTING INSTITUTIONS}

\author{
UNIVERSITY OF BRITISH COLUMBIA \\ CALIFORNIA INSTITUTE OF TECHNOLOGY \\ UNIVERSITY OF CALIFORNIA \\ MONTANA STATE UNIVERSITY \\ UNIVERSITY OF NEVADA \\ NEW MEXICO STATE UNIVERSITY \\ OREGON STATE UNIVERSITY \\ UNIVERSITY OF OREGON \\ OSAKA UNIVERSITY \\ UNIVERSITY OF SOUTHERN CALIFORNIA
}

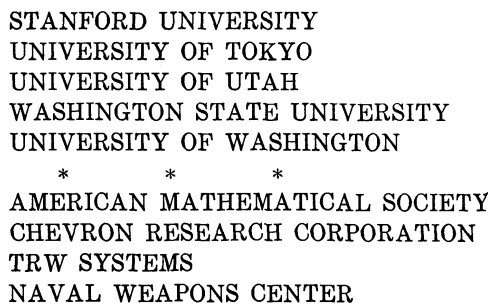

The Supporting Institutions listed above contribute to the cost of publication of this Journal, but they are not owners or publishers and have no responsibility for its content or policies.

Mathematical papers intended for publication in the Pacific Journal of Mathematics should be in typed form or offset-reproduced, double spaced with large margins. Underline Greek letters in red, German in green, and script in blue. The first paragraph or two must be capable of being used separately as a synopsis of the entire paper. It should not contain references to the bibliography. Manuscripts, in duplicate if possible, may be sent to any one of the four editors. Please classify according to the scheme of Math. Rev. 36, 1539-1546. All other communications to the editors should be addressed to the managing editor, Richard Arens, University of California, Los Angeles, California, 90024.

50 reprints are provided free for each article; additional copies may be obtained at cost in multiples of 50 .

The Pacific Journal of Mathematics is published monthly. Effective with Volume 16 the price per volume (3 numbers) is $\$ 8.00$; single issues, $\$ 3.00$. Special price for current issues to individual faculty members of supporting institutions and to individual members of the American Mathematical Society: $\$ 4.00$ per volume; single issues $\$ 1.50$. Back numbers are available.

Subscriptions, orders for back numbers, and changes of address should be sent to Pacific Journal of Mathematics, 103 Highland Boulevard, Berkeley, California, 94708.

PUBLISHED BY PACIFIC JOURNAL OF MATHEMATICS, A NON-PROFIT CORPORATION

Printed at Kokusai Bunken Insatsusha (International Academic Printing Co., Ltd.), 7-17, Fujimi 2-chome, Chiyoda-ku, Tokyo, Japan.

* Acting Managing Editor. 


\section{Pacific Journal of Mathematics}

\section{Vol. 32, No. 3 \\ March, 1970}

Shair Ahmad, Dynamical systems of characteristic $0^{+} \ldots \ldots \ldots \ldots \ldots$

Charles A. Akemann and Bernard Russo, Geometry of the unit sphere of a $C^{*}$-algebra and its dual............................ 575

Philip Bacon, The compactness of countably compact spaces ......... 587

Richard Blaine Barrar and Henry Loeb, On the continuity of the nonlinear Tschebyscheff operator ............................ 593

L. Carlitz, Factorization of a special polynomial over a finite field ....... 603

Joe Ebeling Cude, Compact integral domains .................... 615

Frank Rimi DeMeyer, On automorphisms of separable algebras. II . . . . . 621

James B. Derr, Generalized Sylow tower groups .................. 633

Raouf Doss, Some inclusions in multipliers ................... 643

Mary Rodriguez Embry, The numerical range of an operator........... 647

John Froese, Domain-perturbed problems for ordinary linear differential

operators..................................... 651

Zdeněk Frolík, Absolute Borel and Souslin sets ..................... 663

Ronald Owen Fulp, Tensor and torsion products of semigroups .......... 685

George Grätzer and J. Płonka, On the number of polynomials of an

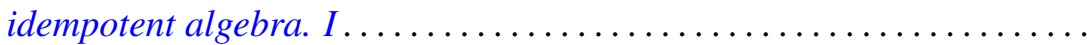

Newcomb Greenleaf and Walter Read, Positive holomorphic differentials on

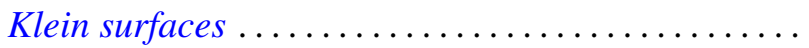

John Willard Heidel, Uniqueness, continuation, and nonoscillation for a second order nonlinear differential equation ................. 715

Leon A. Henkin, Extending Boolean operations................... 723

R. Hirshon, On hopfian groups .......................... 753

Melvin Hochster, Totally integrally closed rings and extremal spaces ..... 767

R. Mohanty and B. K. Ray, On the convergence of a trigonometric integral ..................................

Michael Rich, On a class of nodal algebras .................... 787

Emile B. Roth, Conjugate space representations of Banach spaces ........ 793

Rolf Schneider, On the projections of a convex polytope .............. 799

Bertram Manuel Schreiber, On the coset ring and strong Ditkin sets ...... 805

Edgar Lee Stout, Some remarks on varieties in polydiscs and bounded

holomorphic functions .........................

James Edward Ward, Two-groups and Jordan algebras . 